\title{
Encoding-Based Tamper-Resistant Algorithm for Mobile Device Security
}

\author{
Seok Min Yoon, Seung Wook Lee, Hong Moon Wang, and Jong Tae Kim \\ School of Information and Communication Engineering, \\ Sungkyunkwan University, Korea \\ jtkimeskku.ac.kr
}

\begin{abstract}
Due to the advance in development technology for mobile systems, the attacks on the embedded systems become sophiscated. Especially, tampering with the infomation of mobile devices by software or hardware attack may lead to a serious problem like leaking personal information. In order to protect the tampering attack, encryption technique for embedded information is proposed. However, when the specification of encryption algorithm is known, the system is easily tampered. In this paper, we propose a novel tamperresistant algorithm by encoding program instructions, which can detect the tampering attack. In this algorithm, when a malicious attacker try to tamper with a part of the system, the logical interdepency of program instrutions make it impossible unless he gets the access authorization of whole system.
\end{abstract}

Keywords: Tamper-resistant, Security, Mobile Device.

\section{Introduction}

While technological advances that have improved the development of embedded systems bring the conveniences in human life, it can lead to serious problems: leaking out personal information which stored in a handheld mobile embedded system such as PDAs and smart phones or operating unattempted actions by malicious attacker[1]. The attacks on the embedded systems can be classified in two categories: software attack which exploits implementation flaws by attacking vulnerability of software and hardware attack which exploit the internal information by physical modification or external devices[1]. The hardware attacks which are frequently used on the embedded system are bus tapping and data alteration between processor and external devices. The bus tapping and data alteration, mixed with software vulnerability, may give more ways of breaking a system to a malcious attacker[2][3]. Since the information which saved in a system can be exposed directly by the bus tapping, encryption of internal information techniques are proposed to protect the system[4]. However, Huang proved that the security techniques are neutralized by data which is acquired by tapping system bus exposed externally with simple hardware attachment in case the secret key used in encryption process is known by attacker[5].

In this paper, we propose a tamper-resistant algorithm based on the encoding process of program instructions. The mobile device with the encoding algorithm achieves high security, because it detects both software and hardware attacks effectively by 
checking the interdependencies of instructions[6]. Especially, it can prevent abnormal changes of control routine and execution of malicious program code.

\section{Tampering Detection Algorithm}

A properly working program means that it works as the way intended by developers, that is, the program which does not work as the way intended by developers is not working properly. It means that every program have an execution sequence of instructions which is intended by developers. An instruction executing currently by a processor has interdependencies with a previously executed instruction, and also with a next instruction. If there are abnormal instructions which are not intended by developers in the sequence of execution, interdependencies of instructions are broken. We can, therefore, detect the software and hardware attacks on our system effectively if it is possible to check the integrity of current instruction by examining interdependencies among previous instruction, current instruction and next instruction.

We derive the interdependencies of the instructions by encode current instruction with previous instruction and next encoded code as shown in figure 1 . The encoding and decoding operation of current instruction are defined as below.

$$
\begin{aligned}
& C_{t}=E_{k}\left(I_{t} \text { xor } I_{t-1} \text { xor } C_{t+1}\right) \\
& I_{t}=D_{k}\left(C_{t}\right) \text { xor } I_{t-1} \text { xor } C_{t+1}
\end{aligned}
$$

$\mathrm{E}$ is an encoding function and $\mathrm{D}$ is a decoding function which use cryptography algorithm(ex. DES, AES) with secret key $\mathrm{k}$. The previous instruction and next encoded code are necessary in decoding process, so a processor needs two registers for them.

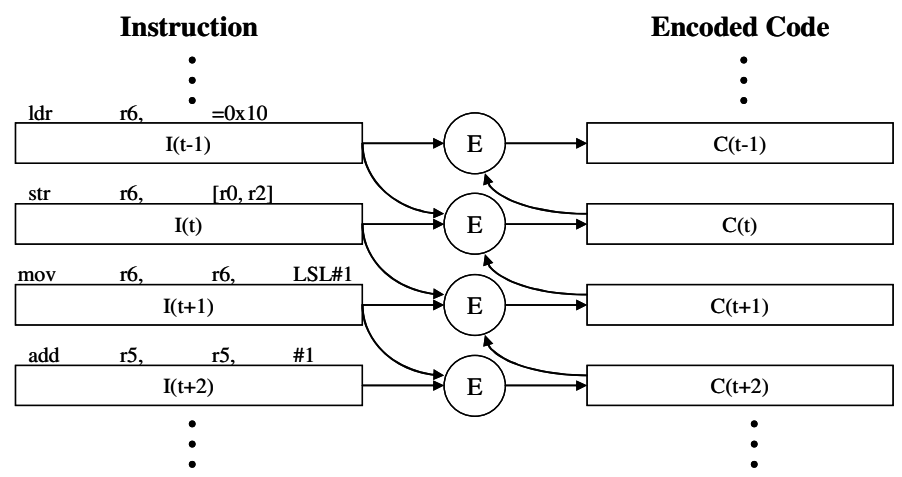

Fig. 1. Encoding process of instructions

\section{Handling of Broken Interdependency}

Basic concept of our algorithm is continuous checking process with the interdependencies of instructions. However, we cannot conclude that every point of broken interdependency is not a point having security problems. In ordinary program, the 
interdependency can be broken by the nature of program itself. The interdependency with a previous instruction cannot be assured at the starting point of a program and the interdependency with a next instruction cannot be assured at the end point of a program. We solve this problem by using magic numbers. Figure 2 shows a simplified program containing $m$ number of instructions. By setting $M_{1}$ as a magic number that only the developers of security process know, the encoding process can be started at the starting point of the program. Similarly, by setting $\mathbf{M}_{2}$ as a parity of whole program, encoding process can be done at the end point.

\begin{tabular}{|c|c|}
\hline Instruction & Encoded Code \\
\hline $\mathrm{M}_{1}$ & \\
\hline $\mathrm{I}_{1}$ & $\mathrm{C}_{1}=\mathrm{E}_{\mathrm{k}}\left(\mathrm{I}_{1}\right.$ xor $\mathrm{M}_{1}$ xor $\left.\mathrm{C}_{2}\right)$ \\
\hline $\mathrm{I}_{2}$ & $\mathrm{C}_{2}=\mathrm{E}_{\mathrm{k}}\left(\mathrm{I}_{2}\right.$ xor $\mathrm{I}_{1}$ xor $\left.\mathrm{C}_{3}\right)$ \\
\hline $\mathrm{I}_{3}$ & $\mathrm{C}_{3}=\mathrm{E}_{\mathrm{k}}\left(\mathrm{I}_{3}\right.$ xor $\mathrm{I}_{2}$ xor $\left.\mathrm{C}_{4}\right)$ \\
\hline $\mathrm{I}_{4}$ & $\mathrm{C}_{4}=\mathrm{E}_{\mathrm{k}}\left(\mathrm{I}_{4}\right.$ xor $\mathrm{I}_{3}$ xor $\left.\mathrm{C}_{5}\right)$ \\
\hline$\ldots$ & $\ldots$ \\
\hline $\mathrm{I}_{\mathrm{m}}$ & $\mathrm{C}_{\mathrm{m}}=\mathrm{E}_{\mathrm{k}}\left(\mathrm{I}_{\mathrm{m}}\right.$ xor $\mathrm{I}_{\mathrm{m}-1}$ xor $\left.\mathrm{M}_{2}\right)$ \\
\hline $\mathrm{M}_{2}$ & \\
\hline
\end{tabular}

Fig. 2. Simplified program

$\begin{array}{lllll}\text { 0x2000000 } & \text { mov } & \text { r5, } & \# 0 & \\ \text { 0x2000004 } & \text { ldr } & \text { r6, } & =0 \times 10 & \\ \text { 0x2000008 } & \text { nop } & & & \\ \text { 0x200000c } & \text { led_loop: } & & & \\ \text { 0x200000c } & \text { str } & \text { r6, } & \text { [r0, r2] } & \\ \text { 0x2000010 } & \text { mov } & \text { r6, } & \text { r6, } & \text { LSL\#1 } \\ \text { 0x2000014 } & \text { add } & \text { r5, } & \text { r5, } & \# 1 \\ \text { 0x2000018 } & \text { cmp } & \text { r5, } & \text { \#loopnum } & \\ \text { 0x200001c } & \text { bne } & \text { led_loop } & & \\ \text { 0x2000020 } & \text { ldr } & \text { r0, } & =0 \times 001 f & \end{array}$

Fig. 3. Insertion of NOP operation

Branch instruction can make a broken interdependency. To prevent this problem, we insert a NOP(No OPeration) instruction at the branch address shown in figure 3 . Whenever a branch instruction is executed, NOP instruction is executed. In general, as the NOP instruction does not have operands, we can save parity as operands for additional comparison process.

\section{Security Analysis}

The encoding and decoding functions use cryptography algorithms. Generally, the key length is very important, because the safety of cryptography algorithms relies on the length of secret key. We assume that the system has 32 bits instruction length, and encoding and decoding operation use 32 bits secret key and the length of encoded code is 32 bits. The 32 bits secret key is fragile on safety because of small key space of $2^{32}$. In order to make up for the weak point, we rearrange the bit composition of encoding input. This has the same effect as increasing the number of cases. Then, the attacker should know the encoding algorithm, the secret key and the bit composition of data used during encoding process. If the attacker know the encoding algorithm, the probability, which the attacker successes on decode, is $3 \times 32 ! \times 2^{32} \approx 2^{151}$ because the attacker should know the secret key, $2^{32}$, and the bit composition of each input, $32 ! \times 32 ! \times 32$ !. In other words, the encoding algorithm has the same effect as using 151 bits secret key.

The basis of our algorithm is logical clearance of the interdependency, not the computational complexity of encoding algorithm. In order to execute current instruction, the integrity of previous instruction, and next instruction should be assured, and 
the interdependency should be guaranteed logically. As we can see in figure 1 and 2, the encoded code $I(t)$ has the interdependencies with previous and next instruction until the end of the program. This means that the interdependencies of whole program are affected by each instruction. In other words, if one instruction has a problem, this problem destroys the interdependencies of whole program.

\section{Conclusion}

In this paper, we proposed an encoding-based tamper-resistant algorithm by checking the interdependency of program instructions as a countermeasure against the malicious attacks on embedded system. The proposed algorithm prevents the system from executing abnormal instructions, when the system is tampered by software or hardware attacks. The encoding algorithm of instruction has the same effect as an cryptography algorithm using 151 bits length of secret key on the system having 32 bits length of instruction. In addition, the logical clearance of interdependency of program instructions makes the attacker impossible to tamper the system unless the attacker gets the access authorization of whole system.

Acknowledgments. This work was supported by the Korea Research Foundation Grant funded by the Korean Government (MOEHRD, Basic Research Promotion Fund) (KRF-2006-521-D00376).

\section{References}

1. S. Ravi, A. Raghunathan, P. Kocher and S. Hattangady, "Security in Embedded Systems: Design Challenges", ACM Transactions on Embedded Computing Systems: Special Issue on Embedded Systems and Security (Guest Editors: D. Serpanos and H.Lekatsas), 2004.

2. X. Zhuang, T. Zhang, and S. Pande. Hide "An infrastructure for efficiently protecting information leakage on the address bus", In Proceedings of the ASPLOS-XI, Oct. 2004.

3. G.E. Suh, D. Clarke, B. Gassend, M.v. Dijk, S. Devadas, "aegis: Architecture for TamperEvident and Tamper-Resistant Processing", In Proceedings of the 17th Int. Conference on Supercomputing, Jun. 2003.

4. G.E. Suh, D. Clarke, B. Gassend, M.v. Dijk, S. Devadas, "Efficient Memory Integrity Verification and Encryption for Secure Processors", Proc. Annual IEEE/ACM Int. Symposium on Microarchitecture (MICRO), pp. 339-350, Dec. 2003.

5. Andrew Huang, "Keeping Secrets in Hardware: The Microsoft XboxTM Case Study", pp. 213 - 227, May, 2002.

6. S. W. Lee and J. T Kim, "Tampering Detection Technique in Instruction Level using Error Detection Code", Lecture Series on Computer and Computational Sciences, Nov. 2005. 